\title{
Moduli spaces in genus zero and inversion of power series
}

\author{
Curtis T. McMullen*
}

\begin{abstract}
This note shows, using elementary properties of ribbon trees, that the universal formula for the inversion of power series can be obtained by counting strata in the compactified moduli space $\overline{\mathcal{M}}_{0, n}$.
\end{abstract}

Mathematics Subject Classification (2010). 32G15.

Keywords. Moduli space, Euler characteristic, stable graphs.

Let $\mathcal{M}_{0, n}$ denote the moduli space of Riemann surfaces of genus 0 with $n$ ordered marked points. Its Deligne-Mumford compactification $\overline{\mathcal{M}}_{0, n}$ is naturally partitioned into connected strata of the form

$$
S \cong \mathcal{M}_{0, n_{1}} \times \cdots \times \mathcal{M}_{0, n_{S}}
$$

indexed by the different topological types of stable curves with $n$ marked points. The stable curves in the stratum above have $s$ irreducible components and $s-1$ nodes; thus $\sum n_{i}=n+2 s-2$.

This note provides a short proof of the following result, which shows that the universal formula for inversion of power series is encoded in the stratification of moduli space.

Theorem 1. The formal inverse of

$$
f(x)=x-\sum_{2}^{\infty} a_{n} x^{n} / n !
$$

is given by

$$
g(x)=x+\sum_{2}^{\infty} b_{n} x^{n} / n !
$$

\footnotetext{
${ }^{*}$ Research supported in part by the NSF.
} 
where

$$
b_{n}=\sum a_{n_{1}} \cdots a_{n_{s}} \times\left(\begin{array}{c}
\text { the number of strata } S \subset \overline{\mathcal{M}}_{0, n+1} \\
\text { isomorphic to } \mathcal{M}_{0, n_{1}+1} \times \cdots \times \mathcal{M}_{0, n_{s}+1}
\end{array}\right) .
$$

That is, $g(f(x))=x$.

Here the coefficients of $f(x)$ and $g(x)$ are regarded as elements of the polynomial ring $\mathbb{Q}\left[a_{2}, a_{3}, \ldots\right]$, and the sum is over all $s \geq 1$ and all multiindices $\left(n_{1}, \ldots, n_{s}\right)$ with $n_{i} \geq 2$.

Using basic properties of the Euler characteristic, we obtain:

Corollary 2 (Getzler). The generating functions

$$
f(x)=x-\sum_{n=2}^{\infty} \chi\left(\mathcal{M}_{0, n+1}\right) \frac{x^{n}}{n !} \text { and } g(x)=x+\sum_{n=2}^{\infty} \chi\left(\overline{\mathcal{M}}_{0, n+1}\right) \frac{x^{n}}{n !}
$$

are formal inverses of one another.

It is easy to see that $a_{n}=\chi\left(\mathcal{M}_{0, n+1}\right)=(-1)^{n}(n-2)$ !, using the fibration $\mathcal{M}_{0, n+1} \rightarrow \mathcal{M}_{0, n}$. Thus by formally inverting $f(x)$, one can readily compute

$$
\left\langle\chi\left(\overline{\mathcal{M}}_{0, n}\right)\right\rangle_{n=3}^{\infty}=\langle 1,2,7,34,213,1630,14747,153946,1821473, \ldots\rangle .
$$

Corollary 2 is a consequence of [Ge1], Theorem 5.9, stated explicitly in [LZ], Remark 4.5.3. The development in [Ge1] uses operads and yields more information, such as Betti numbers for $\overline{\mathcal{M}}_{0, n}$. Theorem 1 shows that Corollary 2 holds for any generalized Euler characteristic on the Grothendieck ring of varieties over $\overline{\mathbb{Q}}$ (cf. [Bi]).

The proof of Theorem 1 will be based on simple properties of trees. Its aim is to provide an elementary entry point to the enumerative combinatorics of moduli spaces.

Trees. A tree $\tau$ is a finite, connected graph with no cycles; its vertices will be denoted $V(\tau)$. The degree function $d: V(\tau) \rightarrow \mathbb{N}$ gives the number of edges incident to each vertex. To each tree we associate the monomial

$$
A(\tau)=\prod_{V(\tau)} A_{d(v)-1}
$$

in the polynomial ring $\mathbb{Z}\left[A_{1}, A_{2}, A_{3}, \ldots\right]$, with the convention $A_{0}=1$.

A tree is stable if it has no vertices of degree 2. An endpoint of $\tau$ is a vertex with $d(v)=1$. We say $\tau$ is rooted if it has a distinguished endpoint (the root). The number of endpoints of $\tau$, other than its root, will be denoted $N(\tau)$. 
We always assume $\tau$ has at least one edge, so $N(\tau) \geq 1$; and the tree with just one edge is considered stable.

A ribbon tree is a rooted stable tree equipped with a cyclic ordering of the edges incident to each vertex. A ribbon structure records the same information as a planar embedding $\tau \hookrightarrow \mathbb{R}^{2}$ up to isotopy.

A marked tree is a rooted stable tree equipped with a labeling of its endpoints by the integers $1,2, \ldots, N(\tau)+1$. We require that the root be labeled 1 .

Theorem 3. The formal inverse of $F(x)=x-\sum_{2}^{\infty} A_{n} x^{n}$ is given by

$$
G(x)=\sum_{\text {ribbon } \tau} A(\tau) x^{N(\tau)} .
$$

Here the sum is taken over all ribbon trees, up to isomorphism.

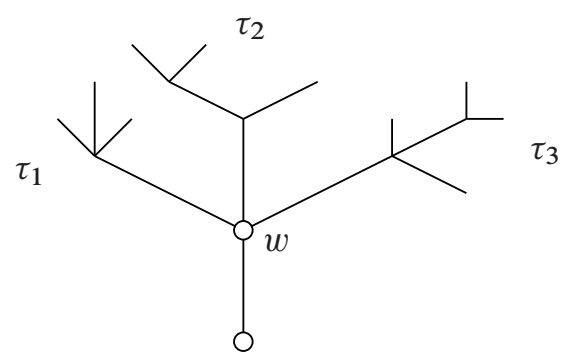

Figure 1

Three ribbon trees grafted together at their roots

Proof. Suppose we are given ribbon trees $\tau_{1}, \ldots, \tau_{d}$ with $d \geq 2$. We can then construct a new ribbon tree $\tau$ by identifying the roots of these trees with a single vertex $w$, and adding a new edge leading from $w$ to the root of $\tau$ (see Figure 1). The ribbon structure at $w$ is determined by the ordering of the trees $\left(\tau_{i}\right)$, and by the condition that the root of $\tau$ lies between $\tau_{d}$ and $\tau_{1}$.

Conversely, any ribbon tree with $N(\tau) \geq 2$ is obtained by applying this construction to the subtrees $\left(\tau_{1}, \ldots, \tau_{d}\right)$ leading away from the edge adjacent to its root. Taking into account the vertex $w$ of degree $d+1$ where these trees are attached, we find:

$$
A(\tau) x^{N(\tau)}=A_{d} \prod_{i=1}^{d} A\left(\tau_{i}\right) x^{N\left(\tau_{i}\right)} .
$$

But the right hand side above is precisely one of the terms occurring in the expression $A_{d} G(x)^{d}$. Summing over all possible values for $d=d(w)$ we obtain

$$
G(x)=x+\sum_{d=2}^{\infty} A_{d} G(x)^{d},
$$


where the first term accounts for the unique tree with $N(\tau)=1$. Rearranging terms gives $F(G(x))=x$.

Corollary 4. The formal inverse of $f(x)=x-\sum_{2}^{\infty} a_{n} x^{n} / n$ ! is given by

$$
g(x)=\sum_{\text {marked }} a(\tau) \frac{x^{N(\tau)}}{N(\tau) !}
$$

where $a(\tau)=\prod_{V(\tau)} a_{d(v)-1}$ and $a_{0}=1$.

Proof. The number of ribbon structures on a given stable rooted tree $\tau$ is given by $\prod(d(v)-1)$ !. The group $\operatorname{Aut}(\tau)$ acts freely on the space of ribbon structures, so $\tau$ contributes $\prod(d(v)-1)$ !/ $|\operatorname{Aut}(\tau)|$ identical terms to equation (1) for $G(x)$. Similarly, $\tau$ contributes $N(\tau)$ !/ $|\operatorname{Aut}(\tau)|$ terms to equation (2) for $g(x)$. Setting $A_{n}=a_{n} / n$ !, we find $F(x)=f(x)$ and

$$
G(x)=\sum_{\text {marked }} \frac{\prod(d(v)-1) !}{N(\tau) !} A(\tau) x^{N(\tau)}=g(x),
$$

so $f(g(x))=F(G(x))=x$.

Remark 1. The same reasoning shows that (2) can be rewritten as

$$
f^{-1}(x)=\sum_{\text {stable } \tau} \frac{N(\tau)+1}{|\operatorname{Aut}(\tau)|} a(\tau) x^{N(\tau)} .
$$

For example, using the trees shown in Figure 2 we find

$$
f^{-1}(x)=x+\frac{a_{2}}{2} x^{2}+\frac{\left(a_{3}+3 a_{2}^{2}\right)}{6} x^{3}+\frac{\left(a_{4}+10 a_{2} a_{3}+15 a_{2}^{3}\right)}{24} x^{4}+O\left(x^{5}\right) \text {. }
$$

For a quite different approach to Corollary 4, see [Ge2], Theorem 1.3.
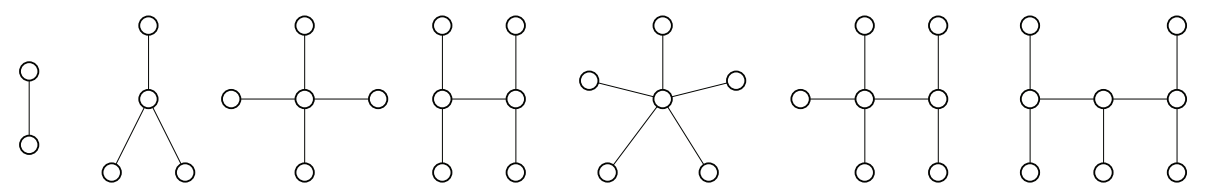

FIGURE 2

The stable trees with $N(\tau) \leq 4$

Proof of Theorem 1. A stable curve $X \in \overline{\mathcal{M}}_{0, n+1}$ of genus zero determines a marked tree $t(X)$ whose interior vertices correspond to the irreducible components of $X$, and whose edges correspond to its nodes and labeled points. Conversely, any marked tree with $N(\tau) \geq 2$ can be realized by a stable curve, so the map

$$
\tau \mapsto S(\tau)=\left\{X \in \overline{\mathcal{M}}_{0, N(\tau)+1}: t(X) \cong \tau\right\}
$$


gives a bijection between marked trees with $N(\tau) \geq 2$ and the strata of moduli spaces. The desired inversion formula now follows from the preceding corollary.

Proof of Corollary 2. Let $a_{n}=\chi\left(\mathcal{M}_{0, n+1}\right)$. It is known that $\chi(X-Y)+\chi(Y)=$ $\chi(X)$ whenever $Y$ is a closed subvariety of a complex variety $X$, see [Ful], p. 141, note 13 , and that $\chi(A \times B)=\chi(A) \times \chi(B)$. The first property implies that $\chi\left(\overline{\mathcal{M}}_{0, n+1}\right)$ is the sum of the Euler characteristics of its strata $S$, and the second implies that

$$
\chi(S)=a_{n_{1}} \cdots a_{n_{S}}
$$

whenever $S \cong \mathcal{M}_{0, n_{1}+1} \times \cdots \times \mathcal{M}_{0, n_{S}+1}$. Thus the stated relationship between generating functions follows from Theorem 1 .

Moduli space over $\mathbb{R}$. The real points of the moduli space form a submanifold $\mathcal{M}_{0, n}(\mathbb{R})$ with $(n-1) ! / 2$ connected components, each homeomorphic to $\mathbb{R}^{n-3}$. Let $M_{n}$ be the component of $\mathcal{M}_{0, n}(\mathbb{R})$ where the marked points can be chosen to lie in $\mathbb{R}$, with $x_{1}<x_{2}<\cdots<x_{n}$. Let $\bar{M}_{n}$ be the closure of $M_{n}$ in $\overline{\mathcal{M}}_{0, n}$. The strata of $\bar{M}_{n}$ are encoded by ribbon trees, since the cyclic ordering of the points $\left(x_{i}\right)$ is preserved under stable limits (cf. [De]). Thus in this setting, Theorem 3 yields:

Corollary 5. The formal inverse of $F(x)=x-\sum_{2}^{\infty} A_{n} x^{n}$ is given by $G(x)=x+\sum_{2}^{\infty} B_{n} x^{n}$, where

$$
B_{n}=\sum A_{n_{1}} \cdots A_{n_{S}} \times\left(\begin{array}{c}
\text { the number of strata } S \subset \bar{M}_{0, n+1} \\
\text { isomorphic to } M_{n_{1}+1} \times \cdots \times M_{n_{S}+1}
\end{array}\right) .
$$

Notes and references. A compendium of results on trees, generating functions and inversion can be found in [St], Chapter 5; see also [Ca]. For background on the many connections between graphs and moduli space, see e.g. [ACG], Chapter XVIII, [LZ], and the references therein.

Acknowledgements. I would like to thank S. Koch for useful conversations related to this note.

\section{References}

[ACG] E. Arbarello, M. Cornalba, and P.A. Griffiths, Geometry of Algebraic Curves. Volume 2. With a contribution by J.D. Harris. Grundlehren der mathematischen Wissenschaften 268. Springer Verlag, Berlin etc., 2011. MR 2807457 Zbl 1235.14002 
[Bi] F. BitTnER, The universal Euler characteristic for varieties of characteristic zero. Compos. Math. 140 (2004), 1011-1032. MR 1086.14016 Zbl 2059227

[Ca] D. Callan, Lagrange inversion and Schröder trees. Preprint 1999. www.stat.wisc.edu/ callan/notes/lagrange_schroder/lagrange_schroder.tex

[De] S.L. Devadoss, Tessellations of moduli spaces and the mosaic operad. In J. P. Meyer, J. Morava and W. S. Wilson (eds.), Homotopy Invariant Algebraic Structures. Contemporary Mathematics 239. American Mathematical Society, Providence, RI, 1999, 91-114. MR 1718078 Zbl 0968.32009

[Ful] W. Fulton, Introduction to Toric Varieties. Annals of Mathematics Studies 131. Princeton University Press, Princeton, NJ, 1993. MR 1234037 Zbl 0813.14039

[Ge1] E. Getzler, Operads and moduli spaces of genus 0 Riemann surfaces. In R. H. Dijkgraaf, C.F. Faber, and G. van der Geer (eds.), The Moduli Space of Curves. Progress in Mathematics 129. Birkhäuser Boston, Boston, MA, 1995, 199-230. MR 1363058 Zbl 0851.18005

[Ge2] — , The semi-classical approximation for modular operads. Comm. Math. Phys. 194 (1998), 481-492. MR 1627677 Zbl 0912.18007

[LZ] S. K. Lando and A. K. Zvonkin, Graphs on Surfaces and Their Applications. With an appendix by Don B. ZAGIER. Encyclopaedia of Mathematical Sciences 141. Low-Dimensional Topology II. Springer Verlag, Berlin, 2004. MR 2036721 Zbl 1040.05001

[St] R.P. Stanley, Enumerative Combinatorics. Volume II. With a foreword by G.-C. Rota and Appendix 1 by S. Fomin. Cambridge Studies in Advanced Mathematics 62. Cambridge University Press, Cambridge, 1999. MR 1676282 Zbl 0928.05001

(Reçu le 2 octobre 2012)

Curtis T. McMullen, Mathematics Department, Harvard University, 1 Oxford Street, Cambridge, MA 02138-2901, U.S.A. 